\title{
A new error estimate on uniform norm of Schwarz algorithm for elliptic quasi-variational inequalities with nonlinear source terms
}

\author{
Allaoua Mehri ${ }^{1 *}$ and Samira Saadi ${ }^{2}$
}

"Correspondence:

allaoua.mehri@yahoo.fr

${ }^{1}$ Lab. LANOS, Department of

Mathematics, University May 8th

1945, Guelma, Algeria

Full list of author information is

available at the end of the article

\section{Springer}

\begin{abstract}
The Schwarz algorithm for a class of elliptic quasi-variational inequalities with nonlinear source terms is studied in this work. The authors prove a new error estimate in uniform norm, making use of a stability property of the discrete solution. The domain is split into two sub-domains with overlapping non-matching grids. This approach combines the geometrical convergence of solutions and the uniform convergence of variational inequalities.
\end{abstract}

MSC: 65N30; 65K15;05C38; 65N15

Keywords: Schwarz algorithm; Overlapping grids; Quasi-variational inequalities; Stability property; Error estimates

\section{Introduction}

In the present paper, we consider the numerical solution of elliptic quasi-variational inequalities with nonlinear right-hand side. This kind of problem has many applications in impulse control (see [1-4]). The existence, uniqueness, and regularity of the continuous and the discrete solution have been studied and established in the past years (see [3-7]). To estimate a new error of the solution, we apply the Schwarz algorithm, so we split the domain into two overlapping sub-domains such that each sub-domain has its own generated triangulations. In this approach we transform the nonlinear problem into a sequence of linear problems in each sub-domain.

To prove the main result of this paper, we construct two discrete auxiliary sequences of Schwarz, and we estimate the error between continuous and discrete Schwarz iterates. The proof is based on a discrete $L^{\infty}$-stability property with respect to both the boundary condition and the source term for variational inequality, while in [8] the proof is based on a stability property with respect to the boundary condition for variational inequality. Regarding research in this domain, for the linear case we refer the reader to [8-12], and for the nonlinear case we refer to [13-15]. The analysis of geometrical convergence of the Schwarz algorithm has been proven in $[8,16,17]$.

This paper consists of two parts. In the first, we formulate the problem of continuous and discrete quasi-variational inequality, we show the monotonicity and stability proper-

(c) The Author(s) 2018. This article is distributed under the terms of the Creative Commons Attribution 4.0 International License (http://creativecommons.org/licenses/by/4.0/), which permits unrestricted use, distribution, and reproduction in any medium, provided you give appropriate credit to the original author(s) and the source, provide a link to the Creative Commons license, and indicate if changes were made. 
ties of discrete solution, then we define the Schwarz algorithm for two sub-domains with overlapping non-matching grids. In the second part, we establish two auxiliary Schwarz sequences, and we prove the main result of this work.

\section{An overlapping Schwarz method for elliptic quasi-variational inequalities with nonlinear source terms}

\subsection{Formulation of the problem}

Let $\Omega$ be an open bounded polygon in $R^{2}$ with sufficiently smooth boundary $\partial \Omega$. We define the bilinear form, for any $u, v \in H^{1}(\Omega)$,

$$
a(u, v)=\int_{\Omega}\left(\sum_{1 \leq i, j \leq 2} a_{i j} \frac{\partial u}{\partial x_{i}} \frac{\partial v}{\partial x_{j}}+\sum_{1 \leq j \leq 2} a_{j} \frac{\partial u}{\partial x_{j}} v+a_{0} u v\right) d x,
$$

the coefficients $a_{i j}(x), a_{j}(x), a_{0}(x)$ are supposed to be sufficiently smooth and satisfy the following conditions:

$$
\begin{aligned}
& \sum_{1 \leq i, j \leq 2} a_{i j}(x) \xi_{i} \xi_{j} \geq \alpha|\xi|^{2}, \quad \xi \in R^{2}, \alpha>0, x \in \bar{\Omega}, \\
& a_{0}(x) \geq \beta>0, \quad \forall x \in \bar{\Omega} .
\end{aligned}
$$

We also suppose that the bilinear form is continuous and strongly coercive

$$
\exists \alpha>0: a(v, v) \geq \alpha\|v\|_{H^{1}(\Omega)}^{2} .
$$

Let the obstacle $M u$ of impulse control be defined by

$$
M u(x)=k+\inf u(x+\xi), \quad x \in \Omega, \xi \geq 0, x+\xi \in \Omega, k>0 .
$$

The operator $M$ maps $L^{\infty}(\Omega)$ into itself and possesses the following properties [1]:

$$
\begin{aligned}
& M u \leq M \widetilde{u}, \quad \text { whenever } u \leq \widetilde{u}, \\
& M(u+c) \leq M u+c, \quad \text { with } c \text { a positive constant }
\end{aligned}
$$

and a closed convex set

$$
K_{g}(u)=\left\{v \in H^{1}(\Omega): v=g \text { on } \partial \Omega, v \leq M u \text { in } \Omega\right\},
$$

where $g$ is a regular function satisfying

$$
g \in W^{2, p}(\Omega), \quad 2 \leq p<\infty .
$$

Let $f(\cdot)$ be the right-hand side supposed nondecreasing and Lipschitz continuous of constant $\sigma$ such that

$$
\sigma / \beta<1
$$


We consider the following elliptic quasi-variational inequality (Q.V.I):

$$
\left\{\begin{array}{l}
\text { find } u \in K_{g}(u) \quad \text { solution of } \\
a(u, v-u) \geq(f(u), v-u), \quad \forall v \in K_{g}(u)
\end{array}\right.
$$

$(\cdot, \cdot)$ denotes the usual inner product in $L^{2}(\Omega)$.

Thanks to [1], the (QVI) (2.11) has a unique solution; moreover, $u$ satisfies the regularity property

$$
u \in W^{2, p}(\Omega), \quad 2 \leq p<\infty .
$$

Let $\tau^{h}$ be a standard regular and quasi-uniform finite element triangulation in $\Omega, h$ being the mesh size. Let $V_{h}$ denote the standard piecewise linear finite element space. The discrete counterpart of (2.11) consists of

$$
\left\{\begin{array}{l}
\text { find } u_{h} \in K_{g}^{h}\left(u_{h}\right) \quad \text { such that } \\
a\left(u_{h}, v_{h}-u_{h}\right) \geq\left(f\left(u_{h}\right), v_{h}-u_{h}\right), \quad \forall v_{h} \in K_{g}^{h}\left(u_{h}\right),
\end{array}\right.
$$

where

$$
K_{g}^{h}\left(u_{h}\right)=\left\{v_{h} \in V_{h} \subset H^{1}(\Omega) / v_{h}=\pi_{h} g \text { on } \partial \Omega, v_{h} \leq r_{h} M u_{h} \text { in } \tau^{h}\right\},
$$

$r_{h}$ is the usual restriction operator in $\Omega$ and $\pi_{h}$ is an interpolation operator on $\partial \Omega$.

Let $\varphi_{i}, i=1,2, \ldots, m(h)$, be basis functions of the space $V_{h}$. We shall assume that the matrix $A$ produced by

$$
A_{i j}=a\left(\varphi_{i}, \varphi_{j}\right)
$$

is $M$-matrix [18].

\subsection{Monotonicity and $L^{\infty}$-stability properties}

We consider the linear case, for example, $f=f(w)$. Let $(f, g),(\widetilde{f}, \widetilde{g})$ be a pair of data of linear functions, and

$$
\xi_{h}=\partial_{h}\left(f, r_{h} M \xi_{h}, \pi_{h} g\right) \in K_{g}^{h}\left(\xi_{h}\right)
$$

is the solution of inequality

$$
a\left(\xi_{h}, v_{h}-\xi_{h}\right) \geq\left(f, v_{h}-\xi_{h}\right), \quad \forall v_{h} \in K_{g}^{h}\left(\xi_{h}\right),
$$

respectively

$$
\widetilde{\xi}_{h}=\partial_{h}\left(\widetilde{f}, r_{h} M \widetilde{\xi}_{h}, \pi_{h} \widetilde{g}\right) \in K_{\tilde{g}}^{h}\left(\widetilde{\xi}_{h}\right)
$$

is the solution of inequality

$$
a\left(\widetilde{\xi}_{h}, v_{h}-\widetilde{\xi}_{h}\right) \geq\left(\widetilde{f}, v_{h}-\widetilde{\xi}_{h}\right), \quad \forall v_{h} \in K_{\widetilde{g}}^{h}\left(\widetilde{\xi}_{h}\right)
$$

Then we give the monotonicity result. 
Lemma 1 Iff $\geq \widetilde{f}, g \geq \widetilde{g}$, then $\partial_{h}\left(f, r_{h} M \xi_{h}, \pi_{h} g\right) \geq \partial_{h}\left(\widetilde{f}, r_{h} M \widetilde{\xi}_{h}, \pi_{h} \widetilde{g}\right)$.

Proof Let us reason by recurrence.

For $n=0$ : let $\xi_{h}^{0}$ (resp. $\left.\widetilde{\xi}_{h}^{0}\right)$ be the solution of equation

$$
\left\{\begin{array}{l}
a\left(\xi_{h}^{0}, v_{h}\right)=\left(f, \xi_{h}^{0}\right), \quad \forall v_{h} \in V_{h}, \\
v_{h}=\pi_{h} g, \quad \text { on } \partial \Omega .
\end{array}\right.
$$

By the maximum principle, we have

$$
\xi_{h}^{0} \geq \widetilde{\xi}_{h}^{0}
$$

and hence by assumption (2.6)

$$
r_{h} M \xi_{h}^{0} \geq r_{h} M \widetilde{\xi}_{h}^{0}
$$

putting

$$
\xi_{h}^{1}=\partial_{h}\left(f, r_{h} M \xi_{h}^{0}, \pi_{h} g\right)
$$

(resp.)

$$
\widetilde{\xi}_{h}^{1}=\partial_{h}\left(\widetilde{f}, r_{h} M \widetilde{\xi}_{h}^{0}, \pi_{h} \widetilde{g}\right),
$$

applying the monotonicity result for (V.I), we get

$$
\xi_{h}^{1} \geq \widetilde{\xi}_{h}^{1}
$$

Now, we define the following sequences:

$$
\xi_{h}^{n}=\partial_{h}\left(f, r_{h} M \xi_{h}^{n-1}, \pi_{h} g\right)
$$

(resp.)

$$
\widetilde{\xi}_{h}^{n}=\partial_{h}\left(\widetilde{f}, r_{h} M \widetilde{\xi}_{h}^{n-1}, \pi_{h} \widetilde{g}\right),
$$

and we assume that

$$
\xi_{h}^{n} \geq \widetilde{\xi}_{h}^{n}
$$

By (2.6), it follows that

$$
r_{h} M \xi_{h}^{n} \geq r_{h} M \widetilde{\xi}_{h}^{n}
$$

therefore, applying again the monotonicity result for (V.I), we obtain

$$
\xi_{h}^{n+1} \geq \widetilde{\xi}_{h}^{n+1}
$$


Finally, if $n \longrightarrow \infty$ (see [1]), we get

$$
\xi_{h} \geq \widetilde{\xi}_{h}
$$

which concludes the proof.

The proposition below establishes an $L^{\infty}$-stability property of the solution with respect to the data.

Proposition 1 Under conditions of Lemma 1, we have

$$
\left\|\partial_{h}\left(f, r_{h} M \xi_{h}, \pi_{h} g\right)-\partial_{h}\left(\tilde{f}, r_{h} M \widetilde{\xi}_{h}, \pi_{h} \widetilde{g}\right)\right\|_{L^{\infty}(\Omega)} \leq \max \left\{\frac{1}{\beta}\|f-\widetilde{f}\|_{L^{\infty}(\Omega)},\|g-\widetilde{g}\|_{L^{\infty}(\partial \Omega)}\right\}
$$

Proof Firstly, set

$$
\Phi=\max \left\{\frac{1}{\beta}\|f-\tilde{f}\|_{L^{\infty}(\Omega)},\|g-\tilde{g}\|_{L^{\infty}(\partial \Omega)}\right\}
$$

we have

$$
a\left(\widetilde{\xi}_{h}, v_{h}-\widetilde{\xi}_{h}\right) \geq\left(\tilde{f}, v_{h}-\widetilde{\xi}_{h}\right)
$$

and

$$
a\left(\Phi, v_{h}-\widetilde{\xi}_{h}\right)=\Phi\left(a_{0}, v_{h}-\widetilde{\xi}_{h}\right)
$$

By summation, we get

$$
a\left(\widetilde{\xi}_{h}+\Phi, v_{h}-\widetilde{\xi}_{h}\right) \geq\left(\tilde{f}+a_{0} \Phi, v_{h}-\widetilde{\xi}_{h}\right)
$$

and

$$
a\left(\widetilde{\xi}_{h}+\Phi,\left(v_{h}+\Phi\right)-\left(\widetilde{\xi}_{h}+\Phi\right)\right) \geq\left(\widetilde{f}+a_{0} \Phi,\left(v_{h}+\Phi\right)-\left(\widetilde{\xi}_{h}+\Phi\right)\right)
$$

If we put

$$
\bar{\xi}_{h}=\widetilde{\xi}_{h}+\Phi, \quad \bar{v}_{h}=v_{h}+\Phi,
$$

then

$$
a\left(\bar{\xi}_{h}, \bar{v}_{h}-\bar{\xi}_{h}\right) \geq\left(\tilde{f}+a_{0} \Phi, \bar{v}_{h}-\bar{\xi}_{h}\right),
$$

therefore

$$
\bar{\xi}_{h}=\partial_{h}\left(\widetilde{f}+a_{0} \Phi, r_{h} M \bar{\xi}_{h}, \pi_{h}(\widetilde{g}+\Phi)\right),
$$


where

$$
\bar{\xi}_{h} \leq r_{h} M \bar{\xi}_{h}=r_{h} M\left(\widetilde{\xi}_{h}+\Phi\right)
$$

By (2.7), it follows that

$$
\widetilde{\xi}_{h} \leq r_{h} M \widetilde{\xi}_{h}
$$

so

$$
\partial_{h}\left(\widetilde{f}+a_{0} \Phi, r_{h} M \bar{\xi}_{h}, \pi_{h}(\widetilde{g}+\Phi)\right)=\partial_{h}\left(\widetilde{f}, r_{h} M \widetilde{\xi}_{h}, \pi_{h} \widetilde{g}\right)+\Phi
$$

Secondly, we have

$$
\begin{aligned}
f & \leq \tilde{f}+\|f-\tilde{f}\|_{L^{\infty}(\Omega)} \\
& \leq \tilde{f}+\frac{a_{0}}{\beta}\|f-\tilde{f}\|_{L^{\infty}(\Omega)} \\
& \leq \tilde{f}+a_{0} \Phi
\end{aligned}
$$

and

$$
\begin{aligned}
g & \leq \tilde{g}+\|g-\tilde{g}\|_{L^{\infty}(\partial \Omega)} \\
& \leq \widetilde{g}+\Phi .
\end{aligned}
$$

Using Lemma 1, we get

$$
\begin{aligned}
\partial_{h}\left(f, r_{h} M \xi_{h}, \pi_{h} g\right) & \leq \partial_{h}\left(\widetilde{f}+a_{0} \Phi, r_{h} M\left(\widetilde{\xi}_{h}+\Phi\right), \pi_{h}(\widetilde{g}+\Phi)\right) \\
& =\partial_{h}\left(\widetilde{f}, r_{h} M \widetilde{\xi}_{h}, \pi_{h} \widetilde{g}\right)+\Phi,
\end{aligned}
$$

then

$$
\xi_{h} \leq \widetilde{\xi}_{h}+\Phi
$$

Similarly, interchanging the roles of the couples $(f, g)$ and $(\widetilde{f}, \widetilde{g})$, we obtain

$$
\widetilde{\xi}_{h} \leq \xi_{h}+\Phi,
$$

which completes the proof.

The following result is due to [6].

Theorem 1 There exists a constant $c$ independent of $h$ such that

$$
\left\|\partial_{h}\left(f, r_{h} M \xi_{h}, \pi_{h} g\right)-\partial(f, M \xi, g)\right\|_{L^{\infty}(\Omega)} \leq c h^{2}|\log h|^{2} .
$$




\subsection{The continuous Schwarz algorithm}

We consider the problem: find $u \in K_{0}(u)$ such that

$$
a(u, v-u) \geq(f(u), v-u), \quad \forall v \in K_{0}(u)
$$

where $K_{0}(u)$ is defined in (2.8) with $g=0$.

We split $\Omega$ into two overlapping polygonal sub-domains $\Omega_{1}$ and $\Omega_{2}$ such that

$$
\Omega_{1} \cap \Omega_{2} \neq \emptyset, \quad \Omega=\Omega_{1} \cup \Omega_{2},
$$

and $u$ satisfies the local regularity condition

$$
u_{\mid \Omega_{i}} \in W^{2, p}\left(\Omega_{i}\right), \quad 2 \leq p<\infty .
$$

We set $\Gamma_{i}=\partial \Omega_{i} \cap \Omega_{j}$, where $\partial \Omega_{i}$ denotes the boundary of $\Omega_{i}$. The intersection of $\Gamma_{1}$ and $\Gamma_{2}$ is assumed to be empty. We will always assume to simplify that $\Gamma_{1}, \Gamma_{2}$ are smooth.

For $w \in C^{0}\left(\bar{\Gamma}_{i}\right)$, we define

$$
V_{i}^{(w)}=\left\{v \in H^{1}\left(\Omega_{i}\right) / v=0 \text { on } \partial \Omega \cap \partial \Omega_{i}, v=w \text { on } \Gamma_{i}\right\}, \quad i=1,2 .
$$

We associate with problem $(2.15)$ the couple $\left(u_{1}, u_{2}\right) \in V_{1}^{\left(u_{2}\right)} \times V_{2}^{\left(u_{1}\right)}$ such that

$$
\begin{aligned}
& \left\{\begin{array}{l}
a_{1}\left(u_{1}, v-u_{1}\right) \geq\left(f\left(u_{1}\right), v-u_{1}\right), \quad \forall v \in V_{1}^{\left(u_{2}\right)}, \\
u_{1} \leq M u_{1}, \quad v \leq M u_{1} \quad \text { in } \Omega_{1},
\end{array}\right. \\
& \left\{\begin{array}{l}
a_{2}\left(u_{2}, v-u_{2}\right) \geq\left(f\left(u_{2}\right), v-u_{2}\right), \quad \forall v \in V_{2}^{\left(u_{1}\right)}, \\
u_{2} \leq M u_{2}, \quad v \leq M u_{2} \quad \text { in } \Omega_{2},
\end{array}\right.
\end{aligned}
$$

where

$$
\begin{aligned}
& a_{i}(u, v)=\int_{\Omega_{i}}\left(\sum_{1 \leq l, j \leq 2} a_{l j} \frac{\partial u}{\partial x_{l}} \frac{\partial v}{\partial x_{j}}+\sum_{1 \leq j \leq 2} a_{j} \frac{\partial u}{\partial x_{j}} v+a_{0} u v\right) d x, \quad i=1,2, \\
& u_{i}=u_{\mid \Omega_{i}}, \quad i=1,2 .
\end{aligned}
$$

Let $u^{0} \in C^{0}(\bar{\Omega})$ be the initial value such that

$$
a\left(u^{0}, v\right)=\left(f\left(u^{0}\right), v\right), \quad \forall v \in H_{0}^{1}(\Omega) .
$$

We define the Schwarz sequence $\left(u_{1}^{n+1}\right)$ on $\Omega_{1}$ such that $u_{1}^{n+1} \in V_{1}^{\left(u_{2}^{n}\right)}$ solves

$$
\left\{\begin{array}{l}
a_{1}\left(u_{1}^{n+1}, v-u_{1}^{n+1}\right) \geq\left(f\left(u_{1}^{n}\right), v-u_{1}^{n+1}\right), \quad \forall v \in V_{1}^{\left(u_{2}^{n}\right)}, \\
u_{1}^{n+1} \leq M u_{1}^{n}, \quad v \leq M u_{1}^{n} \quad \text { in } \Omega_{1},
\end{array}\right.
$$

and respectively $\left(u_{2}^{n+1}\right)$ on $\Omega_{2}$ such that $u_{2}^{n+1} \in V_{2}^{\left(u_{1}^{n}\right)}$ solves

$$
\left\{\begin{array}{l}
a_{2}\left(u_{2}^{n+1}, v-u_{2}^{n+1}\right) \geq\left(f\left(u_{2}^{n}\right), v-u_{2}^{n+1}\right), \quad \forall v \in V_{2}^{\left(u_{1}^{n}\right)}, \\
u_{2}^{n+1} \leq M u_{2}^{n}, \quad v \leq M u_{2}^{n} \quad \text { in } \Omega_{2},
\end{array}\right.
$$


where

$$
\begin{aligned}
& u_{1}^{0}=u^{0} \quad \text { in } \Omega_{1}, \quad u_{2}^{0}=u^{0} \quad \text { in } \Omega_{2}, \\
& u_{1}^{n+1}=0 \quad \text { in } \bar{\Omega} \backslash \bar{\Omega}_{1}, \quad u_{2}^{n+1}=0 \quad \text { in } \bar{\Omega} \backslash \bar{\Omega}_{2} .
\end{aligned}
$$

We give a geometrical convergence theorem (see [8]).

Theorem 2 The sequences $\left(u_{1}^{n+1}, u_{2}^{n+1}\right), n \geq 0$ converge geometrically to the solution $\left(u_{1}, u_{2}\right)$ of system (2.16)-(2.17). More precisely, there exist two constants $0<k_{1}, k_{2}<1$ such that

$$
\begin{aligned}
& \left\|u_{1}-u_{1}^{n+1}\right\|_{L^{\infty}\left(\Omega_{1}\right)} \leq k_{1}^{n} k_{2}^{n}\left\|u^{0}-u\right\|_{L^{\infty}\left(\Gamma_{1}\right)}, \\
& \left\|u_{2}-u_{2}^{n+1}\right\|_{L^{\infty}\left(\Omega_{2}\right)} \leq k_{1}^{n} k_{2}^{n}\left\|u^{0}-u\right\|_{L^{\infty}\left(\Gamma_{2}\right)} .
\end{aligned}
$$

\subsection{The discretization}

Let $\tau^{h_{i}}$ be a standard regular and quasi-uniform finite element triangulation in $\Omega_{i} ; i=1,2$, $h_{i}$ being the mesh size. We assume that $\tau^{h_{1}}$ and $\tau^{h_{2}}$ are mutually independent on $\Omega_{1} \cap \Omega_{2}$, in the sense that a triangle belonging to $\tau^{h_{i}}$ does not necessarily belong to $\tau^{h_{j}}, i \neq j$. Let $V_{h_{i}}=V_{h_{i}}\left(\Omega_{i}\right)$ be the space of continuous piecewise linear functions on $\tau^{h_{i}}$ which vanish on $\partial \Omega \cap \partial \Omega_{i}$. For given $w \in C^{0}\left(\bar{\Gamma}_{i}\right)$, we set

$$
V_{h_{i}}^{(w)}=\left\{v_{h_{i}} \in V_{h_{i}} / v_{h_{i}}=\pi_{h_{i}}(w) \text { on } \Gamma_{i}\right\}, \quad i=1,2,
$$

where $\pi_{h_{i}}$ denotes a suitable interpolation operator on $\Gamma_{i}$. We give the discrete counterpart of the Schwarz algorithm defined in (2.19) and (2.20) as follows.

Let $u_{h_{i}}^{0}=r_{h_{i}} u^{0}$ be given, we define the discrete Schwarz sequence $\left(u_{1 h_{1}}^{n+1}\right)$ on $\Omega_{1}$ such that $u_{1 h_{1}}^{n+1} \in V_{h_{1}}^{\left(u_{2 h_{2}}^{n}\right)}$ solves

$$
\left\{\begin{array}{l}
a_{1}\left(u_{1 h_{1}}^{n+1}, v_{h_{1}}-u_{1 h_{1}}^{n+1}\right) \geq\left(f\left(u_{1 h_{1}}^{n}\right), v_{h_{1}}-u_{1 h_{1}}^{n+1}\right), \quad \forall v_{h_{1}} \in V_{h_{1}}^{\left(u_{2 h_{2}}^{n}\right)} \\
u_{1 h_{1}}^{n+1} \leq r_{h_{1}} M u_{1 h_{1}}^{n} v_{h_{1}} \leq r_{h_{1}} M u_{1 h_{1}}^{n} \quad \text { in } \tau^{h_{1}},
\end{array}\right.
$$

and on $\Omega_{2}$ the sequence $u_{2 h_{2}}^{n+1} \in V_{h_{2}}^{\left(u_{1 h_{1}}^{n}\right)}$ solves

$$
\left\{\begin{array}{l}
a_{2}\left(u_{2 h_{2}}^{n+1}, v_{h_{2}}-u_{2 h_{2}}^{n+1}\right) \geq\left(f\left(u_{2 h_{2}}^{n}\right), v_{h_{2}}-u_{2 h_{2}}^{n+1}\right), \quad \forall v_{h_{2}} \in V_{h_{2}}^{\left(u_{1 h_{1}}^{n}\right)} \\
u_{2 h_{2}}^{n+1} \leq r_{h_{2}} M u_{2 h_{2}}^{n} v_{h_{2}} \leq r_{h_{2}} M u_{2 h_{2}}^{n} \quad \text { in } \tau^{h_{2}},
\end{array}\right.
$$

with

$$
u_{1 h_{1}}^{0}=u_{h_{1}}^{0} \quad \text { in } \Omega_{1}, \quad u_{2 h_{2}}^{0}=u_{h_{2}}^{0} \quad \text { in } \Omega_{2} .
$$

We will also assume that the respective matrices produced by problems (2.21) and (2.22) are $M$-matrices [18].

\section{$3 L^{\infty}$-error analysis}

The aim of this section is to show the main result of this paper. To that end, we start by introducing two discrete auxiliary sequences and prove a fundamental lemma. 


\subsection{Two discrete auxiliary sequences}

For $w_{i h_{i}}^{0}=u_{h_{i}}^{0}$, we define the sequence $w_{1 h_{1}}^{n+1} \in V_{h_{1}}^{\left(u_{2}^{n}\right)}$, discrete solution of V.I

$$
\left\{\begin{array}{l}
a_{1}\left(w_{1 h_{1}}^{n+1}, v_{h_{1}}-w_{1 h_{1}}^{n+1}\right) \geq\left(f\left(u_{1}^{n}\right), v_{h_{1}}-w_{1 h_{1}}^{n+1}\right), \quad \forall v_{h_{1}} \in V_{h_{1}}^{\left(u_{2}^{n}\right)} \\
w_{1 h_{1}}^{n+1} \leq r_{h_{1}} M w_{1 h_{1}}^{n} v_{h_{1}} \leq r_{h_{1}} M w_{1 h_{1}}^{n} \quad \text { in } \tau^{h_{1}}
\end{array}\right.
$$

respectively the sequence $w_{2 h_{2}}^{n+1} \in V_{h_{2}}^{\left(u_{1}^{n}\right)}$ satisfies

$$
\left\{\begin{array}{l}
a_{2}\left(w_{2 h_{2}}^{n+1}, v_{h_{2}}-w_{2 h_{2}}^{n+1}\right) \geq\left(f\left(u_{2}^{n}\right), v_{h_{2}}-w_{2 h_{2}}^{n+1}\right), \quad \forall v_{h_{2}} \in V_{h_{2}}^{\left(u_{1}^{n}\right)} \\
w_{2 h_{2}}^{n+1} \leq r_{h_{2}} M w_{2 h_{2}}^{n} v_{h_{2}} \leq r_{h_{2}} M w_{2 h_{2}}^{n} \quad \text { in } \tau^{h_{2}}
\end{array}\right.
$$

To simplify the notation, we take

$$
\begin{aligned}
& |\cdot|_{1}=\|\cdot\|_{L^{\infty}\left(\Gamma_{1}\right)}, \quad|\cdot|_{2}=\|\cdot\|_{L^{\infty}\left(\Gamma_{2}\right)}, \\
& \|\cdot\|_{1}=\|\cdot\|_{L^{\infty}\left(\Omega_{1}\right)}, \quad\|\cdot\|_{2}=\|\cdot\|_{L^{\infty}\left(\Omega_{2}\right)}, \\
& h_{1}=h_{2}=h, \quad r_{h_{1}}=r_{h_{2}}=r_{h}, \quad \pi_{h_{1}}=\pi_{h_{2}}=\pi_{h} .
\end{aligned}
$$

It is clear that $w_{i h_{i}}^{n}, i=1,2$, is the finite element approximations of $u_{i}^{n}$ defined in (2.19), (2.20), respectively, where $f(\cdot)$ is Lipschitz continuous and $\| f\left(u_{i}^{n} \|_{i} \leq c\right.$ (independent of $n$ ).

The following lemma will play a crucial role in proving the main result of this paper.

Lemma 2 Let $\left(u_{i}^{n+1}\right),\left(u_{i h}^{n+1}\right), i=1,2$, be the respective sequences defined in (2.19), (2.20), (2.21), and (2.22). Then there exists a constant $c$ independent of $h$ and $n$ such that

$$
\left\|u_{i}^{n+1}-u_{i h}^{n+1}\right\|_{i} \leq c(n+1) h^{2}|\log h|^{2}, \quad i=1,2 .
$$

Proof Let $\theta=\sigma / \beta$, under assumption (2.10), we have

$$
\theta<1 .
$$

Let us prove by inductionfor $n=0$ :

$$
\left\|u_{1}^{1}-u_{1 h}^{1}\right\|_{1} \leq\left\|u_{1}^{1}-w_{1 h}^{1}\right\|_{1}+\left\|w_{1 h}^{1}-u_{1 h}^{1}\right\|_{1} .
$$

Applying Theorem 1 and Proposition 1, putting $f=f\left(u_{1}^{0}\right), \widetilde{f}=f\left(u_{1 h}^{0}\right)$, we obtain

$$
\begin{aligned}
\left\|u_{1}^{1}-u_{1 h}^{1}\right\|_{1} & \leq c h^{2}|\log h|^{2}+\max \left\{\frac{1}{\beta}\left\|f\left(u_{1}^{0}\right)-f\left(u_{1 h}^{0}\right)\right\|_{1},\left|u_{2}^{0}-u_{2 h}^{0}\right|_{1}\right\} \\
& \leq c h^{2}|\log h|^{2}+\max \left\{\theta\left\|u_{1}^{0}-u_{1 h}^{0}\right\|_{1},\left|u_{2}^{0}-u_{2 h}^{0}\right|_{1}\right\} .
\end{aligned}
$$

If

$$
\max \left\{\theta\left\|u_{1}^{0}-u_{1 h}^{0}\right\|_{1},\left|u_{2}^{0}-u_{2 h}^{0}\right|_{1}\right\}=\theta\left\|u_{1}^{0}-u_{1 h}^{0}\right\|_{1}
$$


then

$$
\begin{aligned}
\left\|u_{1}^{1}-u_{1 h}^{1}\right\|_{1} & \leq c h^{2}|\log h|^{2}+\theta\left\|u_{1}^{0}-u_{1 h}^{0}\right\|_{1} \\
& \leq c h^{2}|\log h|^{2}+\left\|u_{1}^{0}-u_{1 h}^{0}\right\|_{1} .
\end{aligned}
$$

Making use of an error estimate for elliptic variational equations [19], we obtain

$$
\left\|u_{1}^{1}-u_{1 h}^{1}\right\|_{1} \leq c h^{2}|\log h|^{2}+c h^{2}|\log h| \leq c h^{2}|\log h|^{2},
$$

and if

$$
\max \left\{\theta\left\|u_{1}^{0}-u_{1 h}^{0}\right\|_{1},\left|u_{2}^{0}-u_{2 h}^{0}\right|_{1}\right\}=\left|u_{2}^{0}-u_{2 h}^{0}\right|_{1},
$$

then

$$
\begin{aligned}
\left\|u_{1}^{1}-u_{1 h}^{1}\right\|_{1} & \leq c h^{2}|\log h|^{2}+\left|u_{2}^{0}-u_{2 h}^{0}\right|_{1} \\
& \leq c h^{2}|\log h|^{2}+\left\|u_{2}^{0}-u_{2 h}^{0}\right\|_{2} .
\end{aligned}
$$

Making use again of an error estimate for elliptic variational equations [19], we obtain

$$
\begin{aligned}
\left\|u_{1}^{1}-u_{1 h}^{1}\right\|_{1} & \leq c h^{2}|\log h|^{2}+c h^{2}|\log h| \\
& \leq c h^{2}|\log h|^{2} .
\end{aligned}
$$

Similarly, we have in domain $\Omega_{2}$

$$
\begin{aligned}
\left\|u_{2}^{1}-u_{2 h}^{1}\right\|_{2} & \leq\left\|u_{2}^{1}-w_{2 h}^{1}\right\|_{2}+\left\|w_{2 h}^{1}-u_{2 h}^{1}\right\|_{2} \\
& \leq c h^{2}|\log h|^{2}+\max \left\{\frac{1}{\beta}\left\|f\left(u_{2}^{0}\right)-f\left(u_{2 h}^{0}\right)\right\|_{2},\left|u_{1}^{0}-u_{1 h}^{0}\right|_{2}\right\} \\
& \leq c h^{2}|\log h|^{2}+\max \left\{\theta\left\|u_{2}^{0}-u_{2 h}^{0}\right\|_{2},\left|u_{1}^{0}-u_{1 h}^{0}\right|_{2}\right\} .
\end{aligned}
$$

If

$$
\max \left\{\theta\left\|u_{2}^{0}-u_{2 h}^{0}\right\|_{2},\left|u_{1}^{0}-u_{1 h}^{0}\right|_{2}\right\}=\theta\left\|u_{2}^{0}-u_{2 h}^{0}\right\|_{2}
$$

therefore

$$
\begin{aligned}
\left\|u_{2}^{1}-u_{2 h}^{1}\right\|_{2} & \leq c h^{2}|\log h|^{2}+\theta\left\|u_{2}^{0}-u_{2 h}^{0}\right\|_{2} \\
& \leq c h^{2}|\log h|^{2}+\left\|u_{2}^{0}-u_{2 h}^{0}\right\|_{2} \\
& \leq c h^{2}|\log h|^{2}+c h^{2}|\log h| \\
& \leq c h^{2}|\log h|^{2},
\end{aligned}
$$

and if

$$
\max \left\{\theta\left\|u_{2}^{0}-u_{2 h}^{0}\right\|_{2},\left|u_{1}^{0}-u_{1 h}^{0}\right|_{2}\right\}=\left|u_{1}^{0}-u_{1 h}^{0}\right|_{2}
$$


then

$$
\begin{aligned}
\left\|u_{2}^{1}-u_{2 h}^{1}\right\|_{2} & \leq c h^{2}|\log h|^{2}+\left|u_{1}^{0}-u_{1 h}^{0}\right|_{2} \\
& \leq c h^{2}|\log h|^{2}+\left\|u_{1}^{0}-u_{1 h}^{0}\right\|_{1} \\
& \leq c h^{2}|\log h|^{2}+c h^{2}|\log h| \\
& \leq c h^{2}|\log h|^{2} .
\end{aligned}
$$

Let us now assume that

$$
\left\|u_{1}^{n}-u_{1 h}^{n}\right\|_{1} \leq c n h^{2}|\log h|^{2}
$$

and

$$
\left\|u_{2}^{n}-u_{2 h}^{n}\right\|_{2} \leq c n h^{2}|\log h|^{2} .
$$

Consequently,

$$
\begin{aligned}
\left\|u_{1}^{n+1}-u_{1 h}^{n+1}\right\|_{1} & \leq\left\|u_{1}^{n+1}-w_{1 h}^{n+1}\right\|_{1}+\left\|w_{1 h}^{n+1}-u_{1 h}^{n+1}\right\|_{1} \\
& \leq c h^{2}|\log h|^{2}+\max \left\{\frac{1}{\beta}\left\|f\left(u_{1}^{n}\right)-f\left(u_{1 h}^{n}\right)\right\|_{1},\left|u_{2}^{n}-u_{2 h}^{n}\right|_{1}\right\} \\
& \leq c h^{2}|\log h|^{2}+\max \left\{\theta\left\|u_{1}^{n}-u_{1 h}^{n}\right\|_{1},\left|u_{2}^{n}-u_{2 h}^{n}\right|_{1}\right\} .
\end{aligned}
$$

If

$$
\max \left\{\theta\left\|u_{1}^{n}-u_{1 h}^{n}\right\|_{1},\left|u_{2}^{n}-u_{2 h}^{n}\right|_{1}\right\}=\theta\left\|u_{1}^{n}-u_{1 h}^{n}\right\|_{1},
$$

then

$$
\begin{aligned}
\left\|u_{1}^{n+1}-u_{1 h}^{n+1}\right\|_{1} & \leq c h^{2}|\log h|^{2}+\theta\left\|u_{1}^{n}-u_{1 h}^{n}\right\|_{1} \\
& \leq c h^{2}|\log h|^{2}+\left\|u_{1}^{n}-u_{1 h}^{n}\right\|_{1} \\
& \leq c h^{2}|\log h|^{2}+c n h^{2}|\log h|^{2} \\
& \leq c(n+1) h^{2}|\log h|^{2},
\end{aligned}
$$

and if

$$
\max \left\{\theta\left\|u_{1}^{n}-u_{1 h}^{n}\right\|_{1},\left|u_{2}^{n}-u_{2 h}^{n}\right|_{1}\right\}=\left|u_{2}^{n}-u_{2 h}^{n}\right|_{1},
$$

therefore

$$
\begin{aligned}
\left\|u_{1}^{n+1}-u_{1 h}^{n+1}\right\|_{1} & \leq c h^{2}|\log h|^{2}+\left|u_{2}^{n}-u_{2 h}^{n}\right|_{1} \\
& \leq c h^{2}|\log h|^{2}+\left\|u_{2}^{n}-u_{2 h}^{n}\right\|_{2} \\
& \leq c h^{2}|\log h|^{2}+c n h^{2}|\log h|^{2} \\
& \leq c(n+1) h^{2}|\log h|^{2} .
\end{aligned}
$$

Similarly, we prove the estimate in domain $\Omega_{2}$. 


\section{$3.2 L^{\infty}$-error estimate}

Theorem 3 (Main result) Let $\left(u_{i}^{n+1}\right),\left(u_{i h}^{n+1}\right), i=1,2$, be the respective solutions of (2.19), (2.20), (2.21), and (2.22). Then, for $n$ large enough, there exists a constant $c$ independent of $h$ and $n$ such that

$$
\begin{aligned}
& \left\|u_{i}-u_{i h}^{n+1}\right\|_{i} \leq c h^{2}|\log h|^{3}, \\
& \left\|u_{i}-u_{i h}^{n+1}\right\|_{W^{1, \infty}\left(\Omega_{i}\right)} \leq c h|\log h|^{3} .
\end{aligned}
$$

Proof Let us give the proof for $i=1$. The case $i=2$ is similar.

Indeed, let $k=\max \left(k_{1}, k_{2}\right)$. It follows from Theorem 2 and Lemma 2 that

$$
\begin{aligned}
\left\|u_{1}-u_{1 h}^{n+1}\right\|_{1} & \leq\left\|u_{1}-u_{1}^{n+1}\right\|_{1}+\left\|u_{1}^{n+1}-u_{1 h}^{n+1}\right\|_{1} \\
& \leq k_{1}^{n} k_{2}^{n}\left|u-u^{0}\right|_{1}+c(n+1) h^{2}|\log h|^{2} \\
& \leq\left|u-u^{0}\right|_{1}+c(n+1) h^{2}|\log h|^{2} \\
& \leq c h^{2}|\log h|^{2}+c(n+1) h^{2}|\log h|^{2} .
\end{aligned}
$$

We choose $n$ such that

$$
k^{n} \geq h
$$

then

$$
\left\|u_{1}-u_{1 h}^{n+1}\right\|_{1} \leq c h^{2}|\log h|^{3}
$$

and by inverse inequality, we get

$$
\left\|u_{1}-u_{1 h}^{n+1}\right\|_{W^{1, \infty}\left(\Omega_{1}\right)} \leq c h|\log h|^{3}
$$

which is the desired error estimate.

\section{Conclusion}

In this work, we have established a new approach of an overlapping Schwarz algorithm on non-matching grids for a class of elliptic quasi-variational inequalities with nonlinear source terms. We have obtained a new error estimate in uniform norm which is optimal for these problems. The error estimate obtained contains a logarithmic factor with an extra power of $|\log h|$ than expected. We will see that this result plays an important role in the study of an error estimate for evolutionary problems with nonlinear source terms.

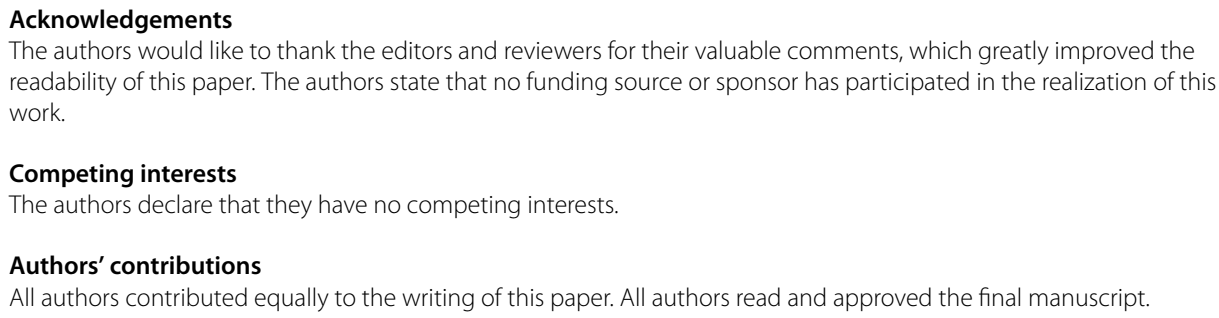




\section{Author details}

${ }^{1}$ Lab. LANOS, Department of Mathematics, University May 8th 1945, Guelma, Algeria. ${ }^{2}$ Lab. LANOS, Department of Mathematics, University Badji Mokhtar Annaba, Annaba, Algeria.

\section{Publisher's Note}

Springer Nature remains neutral with regard to jurisdictional claims in published maps and institutional affiliations.

Received: 29 December 2017 Accepted: 28 February 2018 Published online: 14 March 2018

\section{References}

1. Bensoussan, A., Lions, J.L.: Impulse Control and Quasi-Variational Inequalities. Gauthier-Villars, Paris (1984)

2. Boulbrachene, M.: Pointwise error estimates for a class of elliptic quasi-variational inequalities with nonlinear source terms. Appl. Math. Comput. 161, 129-138 (2005)

3. Cortey-Dumont, P.: Approximation numérique d'une inéquation quasi-variationnelle liée à des problèmes de gestion de stock. RAIRO. Anal. Numér. 14(4), 335-346 (1980)

4. Perthame, B.: Some remarks on quasi-variational inequalities and the associated impulsive control problem. Ann. Inst. Henri Poincaré, Anal. Non Linéaire 2(3), 237-260 (1989)

5. Boulbrachene, M., Saadi, S.: Maximum norm analysis of an overlapping nonmatching grids method for the obstacle problem. Adv. Differ. Equ. 2006, 085807 (2006)

6. Cortey-Dumont, P.: On finite element approximation in the $1^{\infty}$-norm of variational inequalities. Numer. Math. 47, 45-57 (1985)

7. Lions, J.P., Stampacchia, G.: Variational inequalities. Commun. Pure Appl. Math. 20, 493-519 (1967)

8. Saadi, S., Mehri, A.: $1^{\infty}$-error estimate of Schwarz algorithm for elliptic quasi-variational inequalities related to impulse control problem. Aust. J. Math. Anal. Appl. 11(1), 1-13 (2014) http://www.ajmaa.org

9. Badea, L., Tai, X.C., Wang, J.: Convergence rate analysis of a multiplicative Schwarz method for variational inequalities. SIAM J. Numer. Anal. 41(3), 1052-1073 (2003)

10. Boulbrachene, M., Farei, Q.A.: Maximum norm error analysis of nonmatching grids finite element method for linear elliptic PDEs. Appl. Math. Comput. 238, 21-29 (2014)

11. Haiour, M., Boulaares, S.: Overlapping domain decomposition methods for elliptic quasi-variational inequalities related to impulse control problem with mixed boundary conditions. Proc. Indian Acad. Sci. Math. Sci. 121(4), 481-493 (2011)

12. Saadi, S., Mehri, A.: $1^{\infty}$-error estimate of Schwarz algorithm for noncoercive variational inequalities. Appl. Math. 5(3), 572-580 (2014). https://doi.org/10.4236/am.2014.53054

13. Harbi, A., Boulbrachene, M.: Maximum norm analysis of a nonmatching grids method for nonlinear elliptic pdes. J. Appl. Math. 2011, Article ID 605140 (2011)

14. Harbi, A.: Maximum norm analysis of a nonmatching grids method for a class of variational inequalities with nonlinear source terms. J. Inequal. Appl. 2016, 181 (2016) https://doi.org/10.1186/s13660-016-1110-4

15. Zeng, J., Zhou, S.: Schwarz algorithm of the solution of variational inequalities with nonlinear source terms. Appl. Math. Comput. 97, 23-35 (1998)

16. Lions, P.L.: On the Schwarz alternating method. I. In: First International Symposium on Domain Decomposition Methods for Partial Differential Equations, pp. 1-42. SIAM, Philadelphia (1988)

17. Lions, P.L.: On the Schwarz alternating method. II, stochastic interpretation and order proprieties, domain decomposition methods. In: Second International Symposium on Domain Decomposition Methods for Partial Differential Equations, pp. 47-70. SIAM, Philadelphia (1989)

18. Ciarlet, P.G., Raviart, P.A.: Maximum principle and uniform convergence for the finite element method. Comput. Methods Appl. Mech. Eng. 2, 1-20 (1973)

19. Nitshe, J.: $1^{\infty}$-convergence of finite element approximations. In: Proceedings of the Symposium on Mathematical Aspects of Finite Element Methods. Lecture Notes in Mathematics, vol. 606 (1977)

\section{Submit your manuscript to a SpringerOpen ${ }^{\circ}$ journal and benefit from:}

- Convenient online submission

- Rigorous peer review

Open access: articles freely available online

- High visibility within the field

- Retaining the copyright to your article 